\title{
Major MCDM Techniques and their application-A Review
}

\author{
Aarushi Singh ${ }^{1}$, Sanjay Kumar Malik ${ }^{2}$ \\ M. Tech. Student, Hindu College of Engineering, Sonepat \\ Deptt. of CSE, Hindu College of Engineering, Sonepat
}

\begin{abstract}
As the real world problems are so tangled, so the task of decision making associated with them is equally complex. Therefore, some efficient techniques are required which limelight the best solution. For the choice of the optimal selection with respect to the given criteria, this paper has summarized three major and useful techniques of Multiple Criteria Decision Making (MCDM), namely, WSM (Weighted Sum Method), AHP (Analytic Hierarchy Process) and TOPSIS (Technique for Ordered Preference By Similarity to Ideal Solution). The paper highlights the basic steps involved in each of the techniques for choosing the most suitable alternative from among the varied options under consideration.
\end{abstract}

Keywords: - MCDM, WSM, AHP, TOPSIS.

\section{INTRODUCTION}

In order to attain an optimal solution in the presence of so many closely similar options, a strategic move is to be adapted. The alternative are different but since they belong to the same real world set of entity, so the process of the choice of the best among the better options is tricky and requires some degree of in sight.

To optimize the process of the choice, there is a need for the basis which could help differentiating the alternatives and rank the given choices. For doing so, some qualitative and quantitative factors are to be selected which could help in discriminating the alternatives. Such set of factors are collectively known as criteria. For the optimal selection, the alternatives are compared against the selected criteria. Therefore, MCDM is the composition of set of multiple criteria, set of alternatives and their comparison in some manner.

The problem which has been used as a reference in this paper, to describe various techniques- the alternatives are 'the cars' from same or different companies; the criteria include both qualitative as well as quantitative criteria. Qualitative criteria include reliability and style where as quantitative criteria include fuel economy and cost. These are the criteria against which the alternatives have to be compared. The alternative which suits in all the ways is chosen as the best resulting solution. The parameters for optimal choice of the car are as under:-

A. Reliability- Reliability is the probability of failure-free operation of a product for a specified time in a specified environment i.e. for how long the product will work effectively without any failure while the product is under use. So, greater will be the reliability, more will be the probability of failure free life of the product.

B.Style- Style includes the basic appearance, the design and the comfort level of the product. Style directly doesn't affect the quality of the product and of course is a voluntary option. But as the priorities of the masses are being diverted from economical perspective to qualitative perspective, so style is being given greater importance.

C. Fuel Economy- Generally known as mileage, is a basic measure which tells about the fuel consumption per unit distance $(\mathrm{km})$. It reveals how suitable and economical will be the product to the buyer. Lesser is the fuel consumption, higher will be the fuel economy.

D. Cost- Last but not the least rather a major quantitative criteria is the cost. Cost for a car includes actual showroom price, registration price, insurance and accessories price. For a better quality the cost is higher but usually the buyer has a limited budget and therefore the choice is very critical as it is difficult to compromise with the quality also. So, this quantitative factor needs a greater insight. 


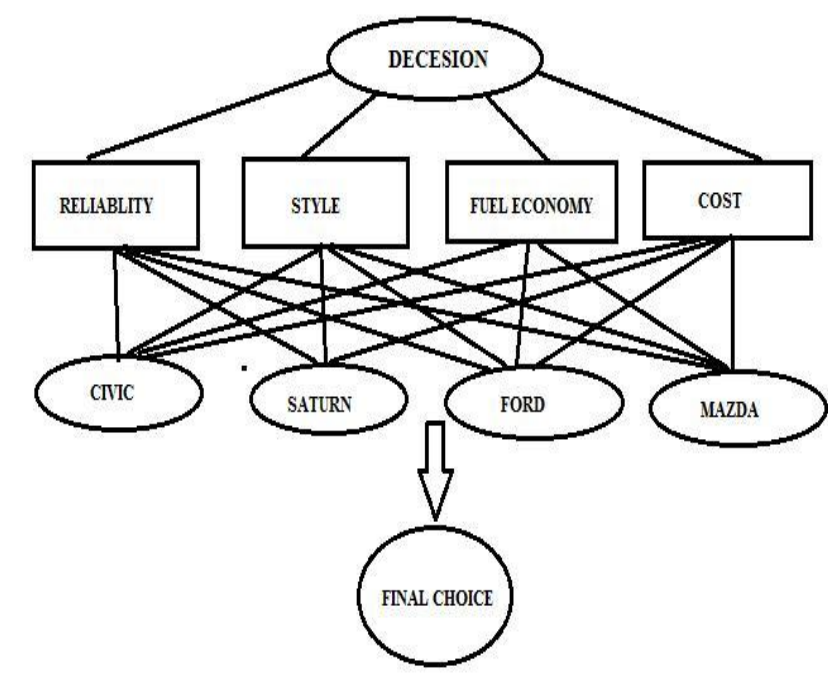

Fig. 1. Hierarchical Structure of MCDM Car Selection Problem

The Fig.1, describes the car selection problem using a hierarchical tree structure. Rectangular boxes represent the criteria against which the alternatives, represented using oval structure are to be compared and final choice has to be done.

\section{LITERATURE REVIEW}

MCDM has a short history of about 40 years, during which this has been an interesting area among the researchers. Over this period, almost 70 MCDM techniques have been explored, [1].

MCDM can be divided into two categories: Multi-Attribute Decision Making (MADM) and Multi-Objective Decision Making (MODM). MADM implicate the selection of the "best" alternative from pre-specified alternatives described in terms of multiple attributes [8]. It is used for the solution of the problems having finite number of alternatives. MODM involves the design of alternatives which optimize the multiple objectives of Decision Maker (DM). The choices are usually infinite or very large and the best will be the one which satiates decision maker's constraints and priorities.

Among the methods that have been evolved out for MCDM, each has varied underlying assumptions, information requirements, analysis models, and decision [9]. This implies that it is critical to select the most appropriate method to solve the problem under consideration, since the use of unsuitable method always leads to misleading decisions. Consequently, incongruous decisions will result in heavy losses. Due to the large warehouse of the MCDM techniques, the selection of appropriate technique is itself a big question to ponder over.

MCDM analysis has some unique characteristics such as the presence of multiple non-commensurable and conflicting criteria, different units of measurement among the criteria, and the presence of quite different alternatives. It is an attempt to review the various MCDM for empirical validation and testing of the various available approaches for the extension of MCDM into group decision-making situations for the treatment of uncertainty [9]. MODM and MADM problems can be further subdivided into two categories depending on the goal preference structure of the decision maker. (i) If there is a single goal-preference structure, the problem is referred to as individual decision making, regardless of the decision makers actually involved (ii). On the other hand, if individuals (interest group) are characterized by different goal-preference structures, the problem becomes that of group decision making [3].

\section{A. Decision Making under Certainty versus Uncertainty}

1. MCDM under certainty: For the decision under certainty it is assumed that all relevant information about the decision situation is known and there is a known deterministic connection between every decision and the corresponding outcome.

2. MCDM under uncertainty: Two basic types of uncertainty may be present in a decision situation. First is the uncertainty associated with limited information about the decision situation and second is the uncertainty associated with fuzziness (impression) concerning the description of the semantic meaning of the events, phenomena, or statements themselves. Consequently, both MODM and MADM problems under uncertainty can be subdivided further into probabilistic and fuzzy decision-making problems, depending on the type of uncertainty involved. 


\section{B. SELECTING MCDM TECHNIQUES}

There is a great diversity in MCDM techniques, this diversity can be seen as a very strong point as well as a weak point too. Diversity facilitates the flexibility in the choice of appropriate technique for a given problem from a wide pool of options but such a huge diverse nature of these techniques makes the appropriate choice more complicated. Each of the technique has its own strengths and weaknesses [9]. In this paper, three techniques have been discussed in detail, lime lighting both their strengths and weaknesses. With reference to the car selection problem, the step by step computation of the choice of the best car has been depicted for each individual technique and the results have been compared mutually. Early in the evolution of MCDM the application of selection techniques for the problems was not considered but now it is clear that consequences of mismatches may lead to suboptimal results, discarding of useful models due to improper application (which means losses in time and money), and finally it may discourage potential users from applying MCDM techniques to real world problems. The WSM is the earliest and probably the most widely used method. The AHP is capable for solving more complex problems and TOPSIS is among the other widely used techniques.

\section{STEPS IN MCDM METHODOLOGY}

MCDM consist of various interrelated steps, that follow one after the other. In this paper, we have tried to present a generic model of MCDM, picturing out the basic concept of the methodologies using series of steps. It is a kind of decision support system which can help in moving along a strategic path to achieve an optimal solution at the end. Following is the Generic MCDM Model which is a flow graph depicting the steps which are essentially included in all the MCDM solutions, followed by the detailed elaboration of each of its step $[2][3][4]$.

\section{Step 1: State and Define the Problem Domain}

The characteristics of the decision making problem under consideration are addressed in the problem definition step, such as identifying the number of alternatives, attributes, and constraints etc. The available information about the decision making problem form the basis of choosing the most appropriate MCDM techniques and will be utilized to solve the problem.

\section{Step 2: Elicit the criteria}

The proper determination of the applicable evaluation criteria is important because they have great influence on the outcome of the MCDM method selection process.

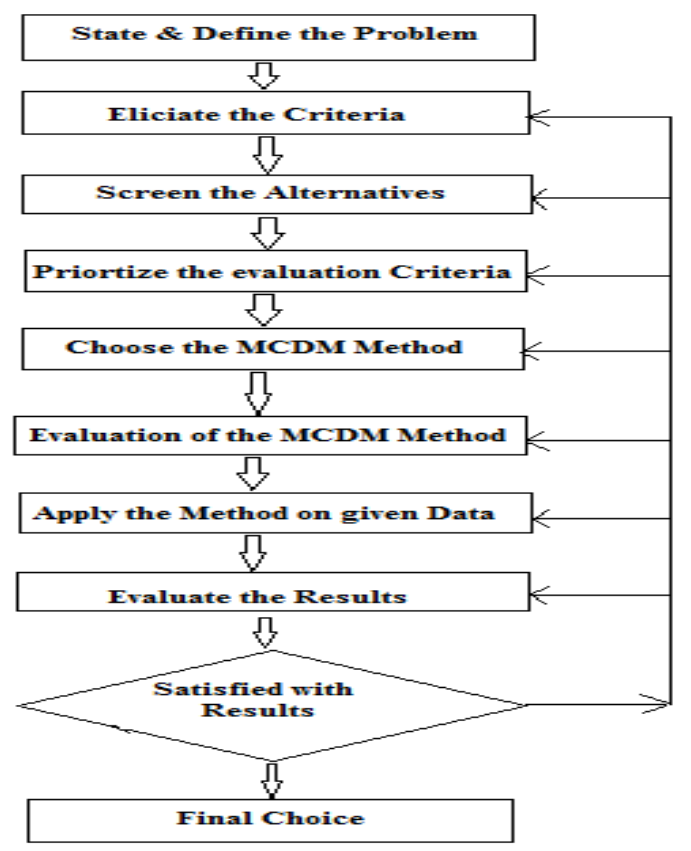

Fig. 2: Generic MCDM Model [2]

However, simply using every criterion in the selection process is not the best approach because the more criteria used, the more information is required, which will result in higher computational cost. The defined evaluation criteria will be used as the attributes of a MCDM formulation and as the input data of decision matrix for method selection. 


\section{Step 3: Screen the alternatives}

An alternative is dominated if there is another alternative which excels it in one or more attributes and equals it in the remainder. The dominated MCDM methods are eliminated by the dominance method, which does not require any assumption or any transformation of attributes. The sieve of dominance takes the following procedures: compare the first two alternatives and if one is dominated by the other, discard the dominated one; then compare the un-discarded alternative with the third alternative and discard any dominated alternative; and then introduce the forth alternative and repeat this process until the last alternative has been compared. A set of non-dominated alternatives may possess unacceptable or infeasible attribute values. The conjunctive method is employed to remove the unacceptable alternatives, in which the DM set up the cut off values he/she will accept for each of the attributes. Any alternative which has an attribute value worse than the cut off values will be eliminated. The cut off values given by the DM play the key role in eliminating the alternatives.

MCDM methods which can perform feasibility evaluation remain as the candidate MCDM methods for further selection.

\section{Step 4: Define the preferences on evaluation criteria}

Usually, after the initial screening step is completed, multiple MCDM methods are expected to remain, otherwise we can directly choose the only one left to solve the decision making problem. This step enforces the prioritization of the criteria. It will help us in identification of the criteria that has the strongest priority and thus will have greatest impact in the final choice and vice-versa.

\section{Step 5: Choose MCDM method for Selection}

This step includes the selection of one of the MCDM method from among the existing commonly used methods. The WSM is chosen as the most suitable MCDM method considering its simplicity and wide generic applicability. Similarly, for complex problems we can opt for the complex techniques. Before the final choice of the method, its cons and pros are necessary to be studied [9].

\section{Step 6: Evaluation of the MCDM method}

The following mathematical formulation, Appropriateness Function (AF) proposed by Li, 2007, is used to rank the MCDM methods. The method with the highest AF, using equation (i), will be recommended as the most appropriate method to solve the problem under consideration.

$$
\begin{aligned}
& A F=\sum w_{i} I_{i} \quad \ldots(i) \\
& I_{i}=\left\{b_{1}, b_{2} \ldots \ldots b_{n}\right\} \\
& b_{i}=1, \quad c_{j i}=a_{i} \quad \text { or } \\
& b_{i}=0, \quad c_{j i} \neq a_{i} \\
& I=1,2 \ldots . . n ; j=1,2 \ldots . . m
\end{aligned}
$$

where $\mathrm{I}$ is the number of evaluation criteria used to examine the decision making methods with respect to the given problem, and $W=W_{1}, W_{2}, W_{3} \ldots \ldots W_{n}$, is the weighting vector on the evaluation criteria, $\mathrm{b}_{\mathrm{i}}$ is the value of the $i^{\text {th }}$ characteristic of the decision problem, and $C_{i j}$ is the value of $i^{\text {th }}$ characteristic of the $j^{\text {th }}$ method. The MCDM method which has the highest AF will be selected as the most appropriate method to solve the given decision making problem.

\section{Step 7:Apply selected Methodology on the Problem}

This step is the inclusion of all the mathematical computations that each of the technique has its own uniquely. In this paper, we have discussed the computations of WSM, AHP and TOPSIS.

\section{Step 8: Results and their evaluation}

The final step is basically the serial outcome of all the above steps and basically of the penultimate step. This paper has analyzed the results of three different techniques. Sensitivity analysis should be performed on the MCDM method selection algorithm in order to analyze its robustness with respect to parameter variations, such as the variation of DM's preference information and the input data.

\section{TECHNIQUES AND THEIR DESCRIPTION}

As described above MCDM is a function of alternatives (available options), criteria (measuring parameters) and their comparison. All the techniques which help in reaching to an optimal state of result are 
more or less a combination of these essential ingredients only.

Following are the three major techniques being discussed highlighting their major strengths, weaknesses and the basic steps.

\section{A. Weighted Sum Method}

The WSM is the one of the earliest and probably the simplest technique that is used in MCDM. Due to its simplicity, the technique is suitable for simple problems, as it basically supports single dimensional problems. WSM allows the comparison of the alternatives by assigning scores, and then using these scores, standard values are generated for the alternatives under consideration. So, overall the results are in the form of good, better and best. The criteria are given weights depending on the severity of each; sum of all these weights must be 1 . Each alternative is assessed with respect to every attribute [5].

\section{PROCESS}

Following generic steps are followed in calculating the relative score for each alternative:-

1) Identify the potential alternatives that can suit best, if chosen.

2) Carefully choose the possible criteria which could be used as the parameters for finding the best alternative.

3) Assign scores to each alternative w.r.t. each criterion.

4) Assign weights to the selected criteria in order to prioritize them.

5) Calculate the total score for each alternative corresponding to all the criteria. This summated score defines the value of a particular alternative.

6) The alternative with the highest score is chosen as the optimal result.

The equation used for calculating the summated score of each alternative is :-

$$
S S_{i}=\sum_{i=1}^{m} w_{i} x_{i j} \quad \text { fori }=1,2 \ldots \ldots n \quad \cdots(i i)
$$

where $S S_{i}$ - Summated Score of $i^{t h}$ alternative for each of the $\mathrm{n}$ alternative.

$W_{j}$ - Weight of the $j^{\text {th }}$ criteria for each of the $\mathrm{m}$ criteria.

$x_{i j}$-Score of the $i^{\text {th }}$ alternative with respect to the $j^{\text {th }}$ criteria.

$\mathrm{n}-$ Number of alternatives.

Initially, it begins up with the construction of a matrix with rows as alternatives and criteria as columns. Each element of the matrix, $X$ define the score of particular alternative against a particular criteria.

\section{Strengths}

- WSM allows well structuring of the problem under consideration, explicitly describing the alternatives, criteria and their relative scores and weights.

- It is a simpler, easy and very suitable approach for solving the multi criteria problems.

- Depiction of the prioritized criteria and the entire computation is comparatively clear and understandable.

\section{Weakness}

- A major drawback which can be visualized in almost all the techniques of MCDM is that the assignment of the weight is a voluntary choice, it not only requires profound insight rather the assignment need to be quite accurate (accuracy itself is a voluntary entity and may differ from problem to problem and situation to situation).

- Weight summation can be accurate if attributes are additive i.e. they must differ from each other in some or the other manner, which may be unrealistic in some cases.

\section{Example}

Now, following is the car selection. There are 3 alternatives, Car A, B, C. The criteria considered are Reliability, Style, Fuel Economy and Cost. The solution required is the final optimal choice that on the basis of these factors, associated weights and the relative scores which can be the best choice of the car. Following is the table defining the scores and the weights:- 
Table 1.Alternatives \& Criteria of the car selection Problem (matrix X)

\begin{tabular}{|l|c|c|c|c|}
\hline Criteria & $\begin{array}{l}\text { Reliability } \\
(0.4)\end{array}$ & $\begin{array}{l}\text { Style } \\
(0.1)\end{array}$ & $\begin{array}{l}\text { Cost } \\
(0.3)\end{array}$ & $\begin{array}{l}\text { Fuel } \\
(0.2)\end{array}$ \\
\hline Car A & 9 & 6 & 6 & 8 \\
\hline Car B & 7 & 7 & 8 & 9 \\
\hline Car C & 9 & 8 & 7 & 8 \\
\hline
\end{tabular}

Following we calculate the summated weighted of each alternate according to equation (ii) starting with Mazda.

$$
\begin{aligned}
& S S_{A}=(0.4 * 7)+(0.1 * 6)+(0.3 * 8)=7.0 \\
& S S_{B}=(0.4 * 9)+(0.1 * 7)+(0.3 * 9)=8.6 \\
& S S_{C}=(0.4 * 7)+(0.1 * 7)+(0.3 * 8)=7.4
\end{aligned}
$$

The maximum score out of all the alternatives is of Car C, hence according to this data and WSM, Car B is considered to be the most optimal choice.

\section{A. The AHP Method}

The AHP technique was actually the result of the research work carried out by Thomas L. Satty in 80 s [6]. With time researchers have produced variants of AHP but in this section of the paper the aim is to highlight the basic procedure of this technique with reference to the car selection problem.

On the whole, the procedure here can be divided into 3 major parts namely, Decomposition of the Problem, Comparative Judgment and Generation of the priorities [5].

Decomposition- As in the WSM, decomposition is associated with breaking the entire problem into its sub parts, in the form of hierarchical structure, thus revealing out the basic structure.

Comparative Judgment - The process of comparative judgment is a two level process. Initially, a matrix of type $A_{N} * N$ is formed in which the relative comparison of alternate with another alternate is done, thus $\mathrm{N}$ here is the number of alternatives. Likewise $\mathrm{M}$ metrics are generated of such form, where $\mathrm{M}$ is the number of criteria under consideration. So, basically this level of comparison consist of $\mathrm{M}$ metrics (one with respect to each criteria) containing the comparison of alternatives among each other. The entries in these matrices, $e_{i j}$ is done according to AHP original measurement scale from one to nine, as given in the following table.

Table 2. Scale of the AHP [6]

\begin{tabular}{|l|l|}
\hline Intensity of Important & Definition \\
\hline 1 & Equal Importance \\
\hline 3 & Moderate Importance \\
\hline 5 & Strong Importance \\
\hline 7 & Very Strong Importance \\
\hline 9 & Extremely Important \\
\hline $2,4,6,8$ & For the intermediate values \\
\hline Reciprocals & $\begin{array}{l}\text { For vice versa Comparison, if } \mathrm{i} \\
\text { to } \mathrm{j} \text { is } 3 \text {, then } \mathrm{j} \text { to i is } 1 / 3\end{array}$ \\
\hline
\end{tabular}

This value is selected basically by the DM defining the relative value (importance) of one alternative when compared with the other keeping one criteria fixed i.e. prioritizing the alternatives on the basis of individual criteria. After the matrix, corresponding to each criterion has been made, it is the time to construct the normalized matrix out of these. A normalized element $r_{i j}$ obtained using equation (iii),

$r_{i j}=\frac{\mathrm{a}_{\mathrm{ij}}}{\sum_{\mathrm{i}=1}^{\mathrm{N}} \mathrm{e}_{\mathrm{ij}}}$

where, $a_{i j}$ is the element of initial matrix $\mathrm{A}_{\mathrm{N} * \mathrm{~N}}$ divided by the sum of all the elements of its respective column. After the normalized matrix for each criteria has been obtained, the weight vector has to be calculated as 
follows, using equation (iv) :-

$$
c=\frac{1}{N \sum_{i=1}^{N} r_{i j}}
$$

where $\mathrm{N}$ is the number of alternatives.

Final step is the formation of an eign vector, which is nothing but ranking of the alternatives according to the particular criteria. Largest value in the eign vector is ranked highest then the smaller then smallest.

Recursively applying the same sequential steps on all the M metrics give the ranking of all the alternative with each possible criteria.

Using the same set of steps, beginning with the initial matrix $C_{M * M}$, (containing the criteria comparison), one more set of eign vector values are generated in addition to the above. Thus, generating the ranking values for the respective criteria too. Though the ranks have been provided to the alternatives as well as to the criteria, but how can one justify that these values are accurate. For this purpose a consistency check has to be performed using the following equation (v) :-

$$
C R=\frac{C I}{R I} \quad \cdots(v)
$$

where, CR is the Consistency Ratio, whose value defines whether the results are consistent or not. CI is the Consistency Index and RI is the Random Index. A perfectly consistent values for CR corresponds to 0 , which is basically an ideal case and that isn't practically feasible. A value of CR which is equal or less than 0.1 indicates acceptable level of consistency in the pair wise matrix, hence acceptable. However, if the value of CR exceeds 0.1 , the comparison values are inconsistent and evaluators need to review their judgments.

\section{CALCULATION OF CONSISTENCY RATIO}

$$
\begin{aligned}
& C R=\frac{C I}{C R} \quad \cdots(v i) \\
& C I=\frac{\lambda_{\text {max }}-n}{n-1} \quad \cdots(\text { vii })
\end{aligned}
$$

The next stage is to calculate $\lambda$ max so as to lead to the Consistency Index and Consistency Ratio.

Step 1: Find the product of A (initial weight matrix) and $x$ (eign vector), $A * x$

Step 2: Lets say, result matrix is Ax. So, now divide Ax by $\mathrm{x}$.

Step 3: Sum up each element of this resulting matrix.

Step 4: This summated value is $\lambda_{\max }$ and $\mathrm{N}$ is the no. of alternatives, hence $\mathrm{CI}$ can be calculated using equation (v).

Step 5: RI depends on the number of elements being compared i.e. N, when alternatives are compared and $\mathrm{M}$ when criteria are compared. A scale has been defined which gives a value of RI corresponding to the no. of elements in comparison.

\section{Example}

Following is a comparison matrix of cars with respect to the criteria "reliability" which will be developed in the car selection problem. All essential steps are described using this matrix.

Table 3: Comparison Matrix of cars w.r.t. Reliability

\begin{tabular}{|l|l|l|l|}
\hline Reliability & Car A & Car B & Car C \\
\hline Car A & 1 & 2 & 8 \\
\hline Car B & $1 / 2$ & 1 & 6 \\
\hline Car C & $1 / 8$ & $1 / 6$ & 1 \\
\hline Column Sum & $13 / 8$ & $19 / 6$ & 15 \\
\hline
\end{tabular}




Table 4: Normalized Matrix
\begin{tabular}{|l|l|l|l|}
\hline Reliability & Car A & Car B & Car C \\
\hline Car A & 0.615 & 0.632 & 0.533 \\
\hline Car B & $1 / 2$ & 0.316 & 0.400 \\
\hline Car C & 0.677 & 0.513 & 0.067 \\
\hline
\end{tabular}

The eign vector ' $\mathrm{x}$ ' obtained using equation (iv) is

$$
\begin{array}{ll}
\text { Car A } & 0.593 \\
\text { Car B } & 0.408 \\
\text { Car C } & 0.264
\end{array}
$$

Now, it is the time to check the consistency of these values. Using the above described steps for calculating, $\lambda$ max, we get 3.019. Using equation (vii) the value of $\mathrm{CI}$ is 0.010 . Using the scale for finding RI, we get $R I=0.58$. Hence, using equation (v) CR is 0.017 . The value is less than 0.1 , hence it is acceptable level of consistent.

The same comparison matrix has to be made for each of the criteria. Same set of operations has to be performed on each matrix, and ranking has to done for each one. After this, Comparison matrix of the criteria is to be made, their relative priority will be measured with its eign vector.

Comparison matrix of alternative with respect to the criteria Cost.

Table 5 : Comparison matrix w.r.t. Cost

\begin{tabular}{|l|l|l|l|}
\hline Cost & Car A & Car B & Car C \\
\hline Car A & 1 & $1 / 3$ & $1 / 4$ \\
\hline Car B & 3 & 1 & $1 / 2$ \\
\hline Car C & 4 & 2 & 1 \\
\hline Column Sum & 8 & $10 / 3$ & $7 / 4$ \\
\hline
\end{tabular}

The eign vector ' $\mathrm{x}$ ' obtained using equation (iii) is
Car A 0.123
Car B 0.320
CarC 0.557

Table 6 : Comparison matrix w.r.t. Fuel Economy.

\begin{tabular}{|l|l|l|l|}
\hline Fuel Economy & Car A & Car B & Car C \\
\hline Car A & 1 & $1 / 4$ & $1 / 6$ \\
\hline Car B & 4 & 1 & $1 / 3$ \\
\hline Car C & 6 & 3 & 1 \\
\hline Column Sum & 11 & $17 / 4$ & $9 / 6$ \\
\hline
\end{tabular}

The eign vector ' $\mathrm{x}$ ' obtained using equation (iii) is Car A 0.087

Car B 0.274

CarC 0.639

Table 7 : Comparison matrix w.r.t. Style.

\begin{tabular}{|l|l|l|l|}
\hline Style & Car A & Car B & Car C \\
\hline Car A & 1 & $1 / 3$ & 4 \\
\hline Car B & 3 & 1 & 7 \\
\hline Car C & $1 / 4$ & $1 / 7$ & 1 \\
\hline Column Sum & $17 / 4$ & $10 / 7$ & 12 \\
\hline
\end{tabular}

The eign vector ' $x$ ' obtained using equation (iii) is

$\operatorname{Car} A \quad 0.265$

Car $B \quad 0.655$

CarC 0.080

Now, when all the alternates have been ranked according to all the criteria then, criteria have to be prioritized using the same process as follows :-

Table 8 : Comparison matrix of criteria

\begin{tabular}{|l|l|l|l|l|}
\hline Criteria & Cost & $\begin{array}{l}\text { Fuel } \\
\text { Economy }\end{array}$ & $\begin{array}{l}\text { Reliabilit } \\
\text { y }\end{array}$ & Style \\
\hline Cost & 1 & 3 & 2 & 2 \\
\hline Fuel Economy & $1 / 3$ & 1 & $1 / 4$ & $1 / 4$ \\
\hline Reliability & $1 / 2$ & 4 & 1 & $1 / 2$ \\
\hline Style & $1 / 2$ & 4 & 2 & 1 \\
\hline
\end{tabular}


Cost $\quad 0.398$

Fuel Economy $\quad 0.085$

Reliability $\quad 0.218$

Style $\quad 0.299$

Finally when everything has been ranked, the answer for the optimal choice is left and that can be deduced by the following way :-

Overall priority of Car A :

$(0.593 * 0.218)+(0.123 * 0.398)+$

$(0.087 * 0.085)+(0.265 * 0.299)=0.265$

Overall priority of Car $B$ :

$(0.408 * 0.218)+(0.320 * 0.398)+$

$(0.274 * 0.085)+(0.655 * 0.299)=0.421$

Overall priority of Car C :

$(0.264 * 0.218)+(0.557 * 0.398)+$

$(0.639 * 0.085)+(0.080 * 0.299)=0.314$

The highest value has been scored by car B. Hence the optimal choice is of Car B.

\section{The TOPSIS Method}

TOPSIS is another technique developed by HWANG and YOON in $80 \mathrm{~s}$, but is being used widely even today. Though the technique has same pet constituents but the principle is quite different The principle of TOPSIS is "The chosen Alternative should have the shortest distance from the ideal solution and the farthest from the negative-ideal solution" Therefore, the method stress on the calculation of the best i.e. the ideal case as well as the worst i.e. negatively ideal case. TOPSIS selects the alternative whose value is closest to the ideal solution and farthest from the negatively ideal solution [7]. Once these values have been found, the optimal case can be generated easily. The major highlights of TOPSIS are :-

1) It is very rational approach where each step of the calculation is very logical and understandable.

2) The calculation involved are simple and straight forward.

3) This technique involves the generation of the ideal and the negative ideal cases, in addition to the generation of most optimal (practically feasible solution).

\section{MAJOR STEPS INVOLVED ARE}

Step 1 - Initially beginning up with the weight matrix, which consists of alternatives, Criteria, weights associated with the criteria and the scores of the alternatives with respect to each of the criteria. ' $\mathrm{M}$ ' are the number of alternative and ' $\mathrm{N}$ ' are the number of criteria So, for the car selection problem, the matrix proceeds as follows where $M=3$ and $N=4$. $X_{i j}$ is the score of $i^{\text {th }}$ alternative with respect to the $j^{\text {th }}$ criteria. So, the final matrix $\mathrm{X}$ for the car selection problem is:-

Table 9: Initial score and weight table for Car selection problem

\begin{tabular}{|l|c|c|c|cc|}
\hline Criteria & $\begin{array}{l}\text { Reliability } \\
(0.4)\end{array}$ & $\begin{array}{l}\text { Style } \\
(0.1)\end{array}$ & $\begin{array}{l}\text { Cost } \\
(0.3)\end{array}$ & $\begin{array}{l}\text { Fuel } \\
(0.2)\end{array}$ & Economy \\
\hline Car A & 9 & 6 & 6 & 8 \\
\hline Car B & 7 & 7 & 8 & 9 \\
\hline Car C & 9 & 8 & 7 & 8 \\
\hline
\end{tabular}

TOPSIS differentiates among the criteria on the basis of their effect and so two different categories have been made namely, $\mathbf{J}$ and $\mathbf{J}$. J consist of the attributes which are to be maximized i.e. Reliability, Style and Fuel 
Economy where as $\mathbf{J}$ comprises of Cost criteria as lesser is the cost more will be the benefit, so cost has to be minimized.

Step 2- Construction of the normalized matrix- The values from the previous matrix are transformed into the form such that their mutual comparison could be done. So, normalization is done as follows yielding out matrix $\mathrm{XN}$ which contain values $x n_{i j}$ :-

$$
Z_{i j}=\frac{X_{i j}}{\left(\sum X_{i j}{ }^{2}\right)^{1 / 2}} \quad \cdots(v i i i)
$$

Table 10: Table showing $\mathrm{x}_{\mathrm{ij}}{ }^{2}, \sum \mathrm{x}_{\mathrm{ij}}{ }^{2},\left(\sum \mathrm{x}_{\mathrm{ij}}{ }^{2}\right)^{1 / 2}$

\begin{tabular}{|l|l|l|l|l|}
\hline & $\begin{array}{l}\text { Reliabil } \\
\text { ity }\end{array}$ & Style & Cost & $\begin{array}{l}\text { Fuel } \\
\text { Econom } \\
\mathrm{y}\end{array}$ \\
\hline Car A & 49 & 36 & 36 & 64 \\
\hline Car B & 81 & 49 & 64 & 81 \\
\hline Car C & 49 & 64 & 49 & 64 \\
\hline$\sum \mathrm{x}_{\mathrm{ij}}{ }^{2}$ & 179 & 149 & 149 & 209 \\
\hline$\left(\sum \mathrm{x}_{\mathrm{ij}}{ }^{2}\right)^{1 / 2}$ & 13.379 & 12.206 & 12.206 & 14.456 \\
\hline
\end{tabular}

Table 10: Normalized Matrix $\left(\mathrm{xn}_{\mathrm{ij}}\right)$

\begin{tabular}{|l|l|l|l|l|}
\hline & Reliability & Style & Cost & $\begin{array}{l}\text { Fuel } \\
\text { Eco. }\end{array}$ \\
\hline $\begin{array}{l}\text { Car } \\
\text { A }\end{array}$ & 0.523 & 0.491 & 0.491 & 0.553 \\
\hline $\begin{array}{l}\text { Car } \\
\text { B }\end{array}$ & 0.672 & 0.573 & 0.655 & 0.622 \\
\hline $\begin{array}{l}\text { Car } \\
\text { C }\end{array}$ & 0.523 & 0.655 & 0.573 & 0.553 \\
\hline
\end{tabular}

Step 3 - This step says that the normalized values now must be multiplied with the weight associated with its corresponding criteria.

Table 11: Multiplication of each column elements with respective criteria weight.

\begin{tabular}{|l|l|l|l|l|}
\hline & Reliability & Style & Cost & $\begin{array}{l}\text { Fuel } \\
\text { Eco. }\end{array}$ \\
\hline Car A & 0.209 & 0.049 & 0.098 & 0.165 \\
\hline Car B & 0.269 & 0.057 & 0.131 & 0.186 \\
\hline Car C & 0.209 & 0.065 & 0.114 & 0.165 \\
\hline
\end{tabular}

Step 4 - Here arises the need to differentiate between the ideal and the negatively ideal solution. For the ideal solution we will determine the highest value for those criteria which are to be maximized (Reliability, Style and Fuel Economy) and the lowest value of the minimizing criteria (Cost) are to be selected. So, this ideal solution is stored in set $I^{*}=\{0.269,0.065,0.098,0.186\}$. On the contrary, for the negatively ideal solution, the choice is just opposite for the criteria. So, the set for negatively ideal solution is $I=\{0.209,0.049,0.131,0.165\}$.

Step-5 : Now we will determine the separation from the ideal solution and separation from the negatively ideal solution by subtracting I* and I' individually from just previous matrix and squaring these values.

Table 12: Separation from ideal solution (subtracting I*)

\begin{tabular}{|c|c|c|c|c|}
\hline & Reliability & Style & Cost & Fuel Eco. \\
\hline $\begin{array}{c}\text { Car } \\
\text { A }\end{array}$ & $(-0.06)^{2}$ & $(-0.016)^{2}$ & $(0)^{2}$ & $(-0.02)^{2}$ \\
\hline $\begin{array}{c}\text { Car } \\
\text { B }\end{array}$ & $(0)$ & $(-0.008)^{2}$ & $(0.033)^{2}$ & $(0)^{2}$ \\
\hline $\begin{array}{c}\text { Car } \\
\text { C }\end{array}$ & $(-0.06)^{2}$ & $(0)^{2}$ & $(-0.017)^{2}$ & $(0)^{2}$ \\
\hline
\end{tabular}

Table 13 : Seperation from negatively ideal solution (subtracting I')

\begin{tabular}{|c|c|c|c|c|}
\hline & Reliability & Style & Cost & Fuel Eco. \\
\hline $\begin{array}{c}\text { Car } \\
\text { A }\end{array}$ & $(0)^{2}$ & $(0)^{2}$ & 0 & $(0)^{2}$ \\
\hline $\begin{array}{c}\text { Car } \\
\text { B }\end{array}$ & $(0.06)^{2}$ & $(-0.008)^{2}$ & $(0.049)^{2}$ & $(0.021)^{2}$ \\
\hline $\begin{array}{c}\text { Car } \\
\text { C }\end{array}$ & $(0)^{2}$ & $(0.016)^{2}$ & $(-0.017)^{2}$ & $(0)^{2}$ \\
\hline
\end{tabular}

Step 6 - Now sum up the values row wise, for all the criteria for 1 particular alternative and then take the under root gives values $\mathrm{T}^{*}$ and $\mathrm{T}^{`}$ for ideal and negatively ideal solution respectively.

For Ideal Solution: 
$\begin{array}{ll}\text { Car } A & \sqrt{0.00040092}>0.02002 \\ \text { Car } B & \sqrt{0.001153}>0.0339 \\ \text { Car } C & \sqrt{0.004256}>0.06523\end{array}$

Step 7 - Last and the final step is the final choice which is done using $C_{i}=\frac{T^{\prime}}{T^{*}-T^{\prime \prime}}$, the highest value of $\mathrm{C}_{\mathrm{i}}$ is the final optimal choice.
Car A $\quad 0.62240$
Car B $\quad 0.82245$
CarC $\quad 0.25807$

So, finally Car B with the highest $C_{i}$ value is the final choice.

\section{MAJOR APPLICATION AREAS FOR MCDM}

MCDM can be applied in all the areas of research and selection in the fields of management, manufacturing, planning, education, transportation, construction, logistic, medical, control and agriculture. MCDM is used in these areas for selection, ranking and evaluation [4][9][10][11].

\section{CONCLUSION AND FUTURE WORK}

MCDM is can be applied anywhere anytime where the DM faces complexity in making a choice. Ranging from the everyday problems and till complex scientific issues, MCDM methodologies can be employed undoubtedly. It has become a powerful tool that can make the process of choice not only easier, but also accurate.

Till date there have been so many methodologies explored and still there is lot to do. It can not only include new application areas but at a higher level a new efficient, fast and practically compatible technique can be evolved out.

Particularly, highlighting the major research areas of computer science, it can be used in Software engineering, Networking, Robotics, Graphics etc.

\section{REFERENCES}

[1] Mohamad Ashari Alias, Siti Zaiton Mohd Hashim and Supiah Samsudin, "Multi Crteria Decision Making And Its Applications: A Literature Review", Asia-Pacific Journal of Information Technology and Multimedia, Jurnal Teknologi Maklumat, Vol 20, No. 2, 2008.

[2] Xiaoqian Sun and Yongchang Li, “An Intelligent Multi-Criteria Decision Support System for Systems Design" $10^{\text {th }}$ AIAA, Aviation Technology, Integration \& Operation Conference, Chapter: 10.2514/6, 2010.

[3] A.E. Dooley, G.W. Sheath and D. Smeaton, "Multiple Criteria Decision Making: Method Selection And Application To Three Contrasting Agricultural Case Studies", NZARES Conference, 2005.

[4] Athanasios C. Karmperis, Konstantinos Aravossis, Ilias P. Tatsiopoulos and Anastasios Sotirchos, "Decision support models for solid waste management: Review and game-theoretic approaches", Waste Management, ELSEVIER, 2013

[5] Milan Janic and Aura Reggiani, "An Application of the Multiple Criteria Decision Making (MCDM) Analysis to the Selection of a New Hub Airport", European Journal of Transport and Infrastructure Research, Vol 2, pp. 113 - 142, 2002.

[6] Saaty TL. The analytic hierarchy process. New York: McGraw- Hill, 1980.

[7] Pema Wangchen Bhutia and Ruben Phipon, "Appication Of Ahp And Topsis Method For Supplier Selection Problem", IOSR Journal of Engineering, Volume 2, Issue 10, pp. 43-50, 2012.

[8] R.V. Rao, "Introduction to Multiple Attribute Decision-making (MADM) Methods", Decision Making in the Manufacturing Environment, Springer Series in Advanced Manufacturing, pp 27-41, 2007.

[9] Martin Aruldoss, T. Miranda Laxmi and V. Prasanna Venkatesan, "A Survey on Multi Criteria Decision Making Methods and Applications", Science and Education Publishing, Issue 1, Vol 1, pp. 31-43, 2013.

[10] Edward W. N. Bernroider \& Johann Mitlöhner, "Characteristics of the Multiple Attribute Decision Making Methodology in Enterprise Resource Planning Software Decisions”, Communications of the IIMA, Volume 5 Issue 1,pp: 49-58, 2005.

[11] Prince Agarwal, Manjari Sahai, Vaibhav Mishra, Monark Bag and Vrijendra Singh, "A review of multi-criteria decision making techniques for supplier evaluation and selection", International Journal of Industrial Engineering Computations 2, pp: 801-810, 2011 